\title{
Comparison of inductive shearography and thermography for flaw detection in structural adhesives on ideal and application-oriented specimen
}

\author{
by I. Kryukov*, M. Kahlmeyer*, S. Böhm*
}

\begin{abstract}
* Department for Cutting and Joining Manufacturing Processes (tff), University of Kassel, Kurt-Wolters-Straße 3,
\end{abstract} 34125 Kassel, Germany, i.kryukov@uni-kassel.de

\begin{abstract}
Adhesive bonding has become increasingly important for industrial appliances. One reason for this is the increasing demand to join different materials in lightweight constructions of materially optimized designs. However, bonding processes need a high degree of supervision. Therefore, non-destructive testing methods of short duration need to be applied. This brings the methods shearography and active thermography into focus.

This paper aims to compare eddy current shearography and thermography for the non-destructive testing of structural bonds. Different defects are examined in a transmission arrangement. The study shows the results for defect detection in ideal as well as application-oriented testing samples.
\end{abstract}

\section{Introduction}

In contrast to many other joining techniques, bonding has continuously become important as it offers many advantages over other joining techniques such as a planar force transmission and the possibility to join different materials of varying thickness. However, this joining technique has not yet developed its full potential. One reason is that the sensitive bonding process shows a susceptibility towards flaws and defects, thus making quality assurance measures a prerequisite. A $100 \%$ control is often necessary for bonds with highly security relevance. In addition, this type of control is used, when bonding is used at the beginning of a complex process chain or for joints, which are closely related to the creation of value. [1]

Economically, only non-destructive testing methods are suitable for the quality control of bonds. In many cases, established methods such as ultrasound and radiographic testing allow a highly precise analysis of inhomogeneities and defects [2]. However, the use of these methods on an industrial scale is implausible, both financially and organizationally.

Methods such as thermography and shearography can be applied at exactly this point $[3,4]$. They function contactfree, are cheap and can be integrated much easier into production processes due to their highly sensitive, quick, robust and reproducible properties as well as their image producing modes. Whilst active thermography disturbs the thermal balance of the testing sample and examines the thermal diffusivity, shearography evaluates the deformation gradient, as a reaction to stress, of the surface of the testing sample. Local deviances in thermal diffusivity or of the deformation gradient indicate defects in the adhesive seam $[5,6,7]$.

The special advantage offered by thermography is the possibility to identify damages caused by delamination in big and complex components rapidly and non-destructively [8]. Shearography has already shown that thermoelastic effects can be quantified precisely, economically and non-destructively. In addition, this method also allows to draw indirect conclusions on adhesive properties in the bonded material compound. [5,7]

Currently, the individual methods cannot identify all relevant flaws and defects. Therefore, this paper would like to address the question as to whether a coupling of thermography and shearography with their divergent physical properties can broaden the range of defect detection. Using exemplary testing samples, Hung et al. have already proven that missing adhesive and delaminations can be detected in the adhesive seam with shearography and thermography. In this case, different kinds of excitations were used for each testing method (e.g. mechanical excitation for shearography and thermal excitation for thermography). This complicated the combination of the methods and the evaluation of the data. [4,6]

Hence, this study will apply a short-time thermal excitation through inductive heating for both testing methods. Thus, measurements can take place with similar excitation parameters, which, consequently, offers great advantages as regards measurement period und effort when integrating this method into industrial processes. This study will examine several defects of adhesive bonds on the basis of ideal as well as application-oriented testing samples.

\section{Testing sample geometry}

Testing samples with simulated defects were produced for the tests. For the ideal testing sample, the material of the upper joining partner was an aluminum alloy (EN AW 6082, $2 \mathrm{~mm}$ thickness), that of the lower substrate was deep drawing steel (DC04, $1 \mathrm{~mm}$ thickness). The substrates had a length of $120 \mathrm{~mm}$ and width of $50 \mathrm{~mm}$. The materials were joined with an over-lapping single lap bonded joint (over-lapping length $20 \mathrm{~mm}$ ) with an adhesive area of $120 \mathrm{~mm} \times 20 \mathrm{~mm}$.

For the structural adhesive bond, a 2C-PUR adhesive was applied with an adhesive layer thickness of $0.25 \mathrm{~mm}$ (e-modulus $1000 \mathrm{MPa}$, shear strength $23 \mathrm{MPa}$, elongation at break $17 \%$, shore $\mathrm{D}$ hardness 65, glass transition 
temperature $48^{\circ} \mathrm{C}$, temperature stability up to $+125^{\circ} \mathrm{C}$ ). Glass balls with a diameter of $0.25 \mathrm{~mm}$ were inserted in order to achieve a constant thickness of the adhesive layer.

The different types of defects, which were relevant for this study, were spread over an area of $10 \mathrm{~mm}$ along the entire width of the bonding seam (c.f. figure 1). Testing samples for the defect irregular thickness of the adhesive layer were an exception to this design. Here, the thickness of the adhesive increased continuously over the entire length of the bonding seam from a thickness of about $0 \mathrm{~mm}$ to $0.25 \mathrm{~mm}$.

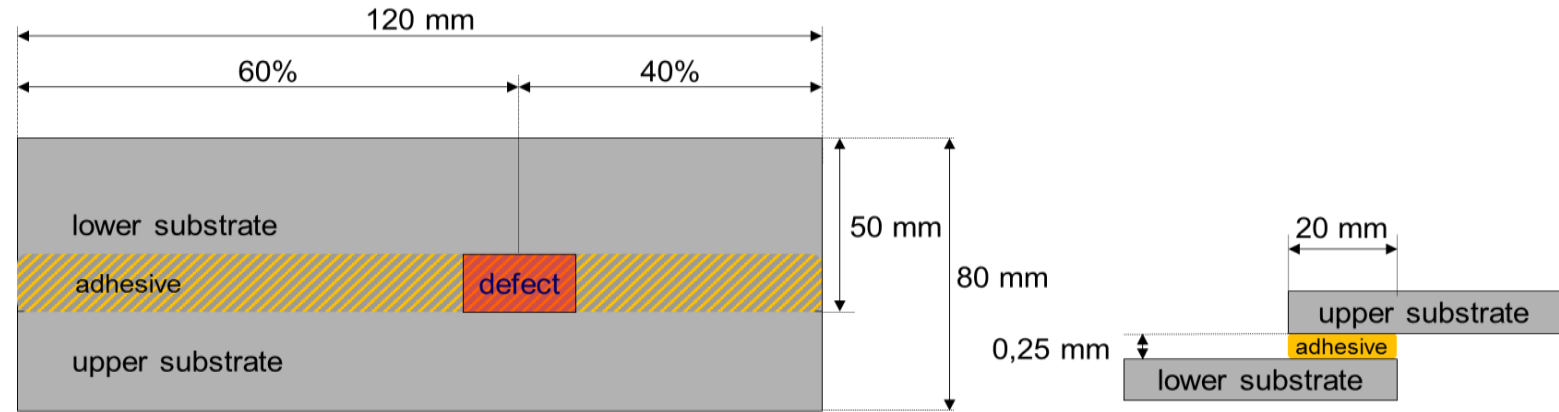

Fig. 1. Schematic drawing and cross section of the geometry of the ideal testing sample as well as the positions of the inserted defects

Seven types of realistic defects were defined and created reproducibly. To give evidence of these defects, destructive testing was performed after non-destructive testing. Flawlessly bonded testing samples were used as reference. The examined types of defects were missing adhesive, voids, irregular thickness of the adhesive, inhomogeneous stirring of adhesive components or varying of the adhesive properties, incorrect mixing ratio or no curing, delamination in form of detachments of the adhesive from the substrate and kissing bonds in form of touching but not firmly bonded adhesive layer.

In order to create uniformly diffuse reflections of the coherent illumination during shearographic measurements as well as to reduce the reflection from the metallic surface of the substrate during thermographic measurements, all testing samples were covered with white top coating.

In accordance to realistic industrial settings, an application-oriented testing sample with a hat profile was also examined. Here, a preformed component made of aluminum (thickness of wall $1.2 \mathrm{~mm}$ ) was used. The component had a length of $400 \mathrm{~mm}$ and a width of $115 \mathrm{~mm}$. On one side, the component showed a characteristic indentation. As counterpart, a sheet of deep drawing steel (thickness of wall $1 \mathrm{~mm}$ ) was added. Both adhesive surfaces of the hat profiles had a width of $18 \mathrm{~mm}$ and a length of $400 \mathrm{~mm}$. In both cases, the same 2C PUR adhesive with an identical adhesive thickness of $0.25 \mathrm{~mm}$ was used. Figure 2 offers a depiction of the adhesively bonded hat profile as seen from above.

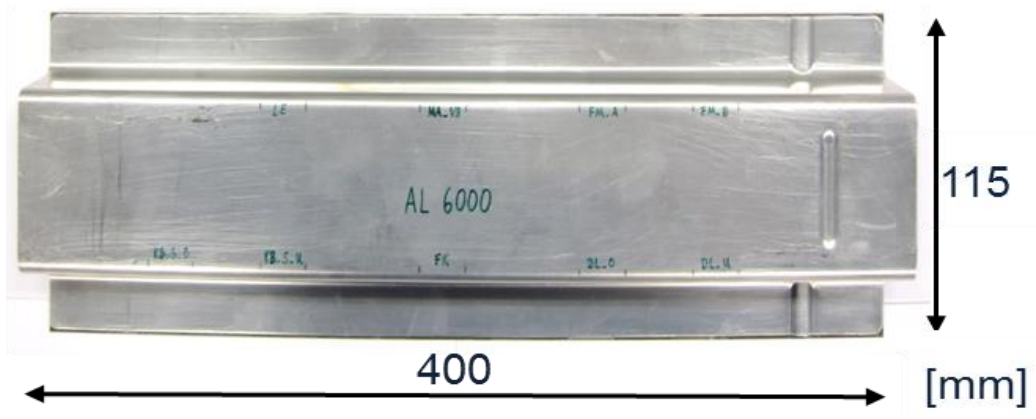

Fig. 2. Top view of the application-oriented testing sample

Defects were inserted into the adhesive layer during bonding. The area in which the defects were positioned had a width of $20 \mathrm{~mm}$. Examined types of defects were missing adhesive, voids, inhomogeneous mixing, wrong mixing ratio (no resin or hardener), delamination (both from hat profile component and counterpart) and kissing bonds (referring to both the hat profile component and counterpart). A specific area was produced without flaws as reference. The positions of the defects are shown in figure 3.

The set measuring field is also depicted in figure 3. Only the area above the upper and the lower bonded seam was evaluated. In order to simplify the transfer of the results to industrial applications, a white coating, as is often applied for shearografic measurements, was omitted. 


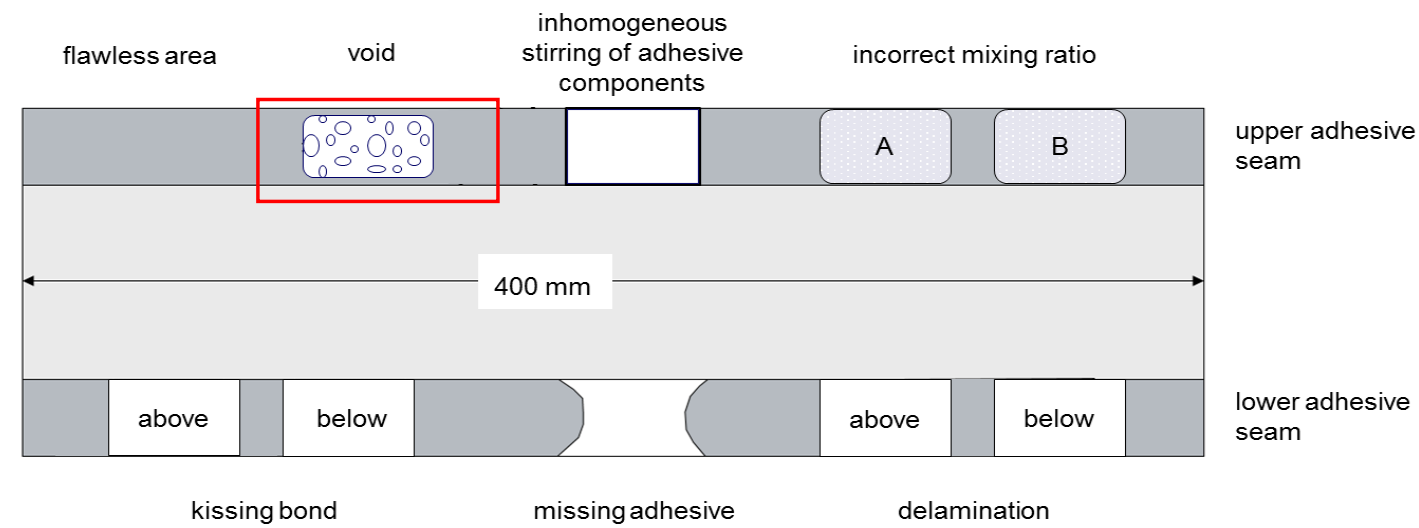

Fig. 3. Schematic depiction of the application-oriented testing sample, including the positions of the defects and the size of the field of view (red frame)

\section{Test Set-Up}

Shearographic measurements were performed using a SE2-sensor with a 5-megapixel CCD-chip from the company isi-sys $\mathrm{GmbH}$. Laser diodes with a wavelength of $658 \mathrm{~nm}$ were used as source for coherent illumination. As shear parameters, an angle of $45^{\circ}$ and a shear distance of $3 \mathrm{~mm}$ were chosen.

FLIR SC5600-M was used for thermographic measurements. The MWIR camera (spectral range $2.5-5.1 \mu \mathrm{m}$ ) had a NETD of $<20 \mathrm{mK}$ and a temperature calibration between $5^{\circ} \mathrm{C}$ and $1500{ }^{\circ} \mathrm{C}$. Measurements were performed with full resolution (640 pixel x 512 pixel) with a recording frequency of $100 \mathrm{~Hz}$ and an integration time of $1 \mathrm{~ms}$.

A coaxial inductor with an induction area of $25 \mathrm{~mm} \times 110 \mathrm{~mm}$ produced the inductive excitation for the homogenous heating of the entire bonding surface. The induction generator had an output of $5 \mathrm{~kW}$. With this system configuration, induction frequencies between $12.5 \mathrm{kHz}$ and $30 \mathrm{kHz}$ could be realized.

Figure 4 shows the test set-up used for the shearographic and thermographic measurements.
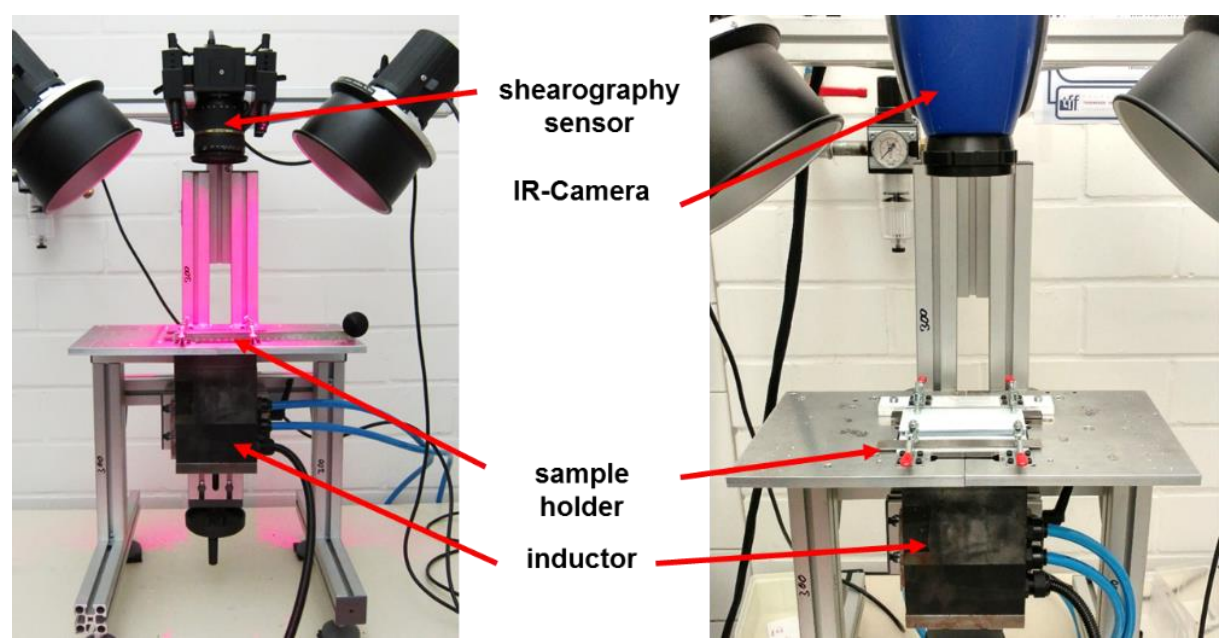

Fig. 4. Depiction of the test set-up of both measurement systems with sample holder and inductor

For testing, the ideal testing samples were fixated into the apparatus and then aligned in accordance to the respective measurement system. Repulsive forces, caused by the Lorentz force during inductive excitation, effect the electrical conductivity, which then needs to be compensated. Therefore, fixation prevented the testing samples from moving during measurements so that these were not disturbed. For this purpose, 4 clamps, connected by 2 connecting clamps, were applied (c.f. figure 5). Apart from preventing any movement of the testing sample and serving to keep the coupling distance of $0.2 \mathrm{~mm}$ to the inductor, this also served to reduce the total distortion of the testing samples and to increase the overall detectability of the defects.

The measurement area for the ideal testing sample is marked with a red frame in figure 5 . The measurement window was defined in such a manner that the boundary of the measurement area was at the upper edge of the upper substrate, both in the shearographic and the thermographic image. The area above the bonded seam as well as a part of the upper substrate were the focus of evaluation. 

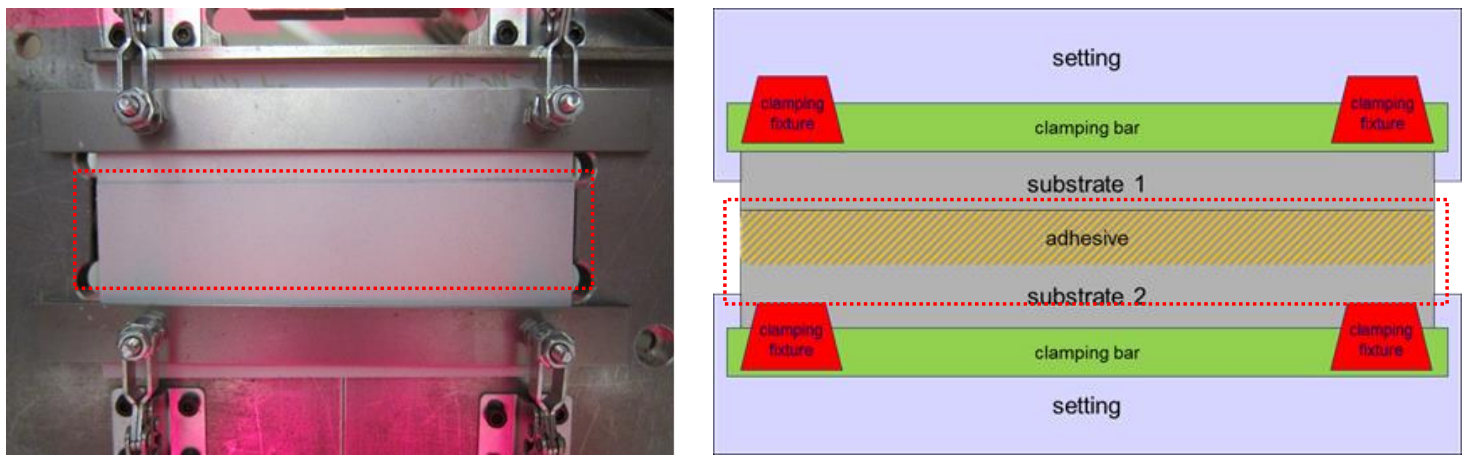

Fig. 5. Fixation of the ideal testing sample with the specimen holder in the measuring field (red frame), left: photo, right: schematic drawing

\section{Detection of Defects in Ideal Testing Samples via Inductively Induced Shearography and Thermography}

Shearographic testing was performed first, followed by thermographic testing. Here, the ideal testing samples were first tested in a position in which the aluminum substrate was set to face the inductor, thus positioning the steel substrate towards the measurement system. Then, the testing samples were turned and measured in the opposing position. This allowed to evaluate the influence of the position of the sample on measurements.

As induction parameters, the induction duration was set at $0.1 \mathrm{~s}$ and the induction frequency at $12.5 \mathrm{kHz}$ in order to achieve the maximum penetration depth for this system configuration [9]. These parameters were kept constant for the combination of both testing methods during the testing series. During shearographic measurements, the set pulse width modulation (PWM, used amount of the pulse range which results from the induction frequency) was $200 \%$. This was increased to $525 \%$ (excitation of the deep drawing steel) and $750 \%$ (excitation of the aluminum substrate) during thermographic measurements - this was the maximum parameter for this technical system. By applying these induction parameters, damage in the adhesive layer could be avoided due to low heating (increase of temperature by $25 \mathrm{~K}$ when heating the deep drawing steel and $2 \mathrm{~K}$ when heating the aluminum). With regard to the option of industrial application, the maximum testing period was limited to 10 seconds for both methods.

For the evaluation of the shearographic measurements, high and low pass filters were applied (e.g. $3 \times 3,7 \times 7$ ). When necessary, demodulation was also performed (also named unfolding; correction of the phase shifts contained in the shearograms).

Pulse-phase thermography was used for thermographic measurements. After the Fourier transformation, the evaluation was performed via phase images with a frequency of $0.1 \mathrm{~Hz}$. This frequency offers the best contrast in the thermographic images. The color scale was adapted to make the detection of the adhesive seam and the contained defects as easy as possible.

Reference measurements using shearography and thermography were performed on flawless testing samples as reference for the evaluation of the flaws and defects (c.f. figure 6). Deviances from the reference measurements indicated irregularities in the adhesive seam.
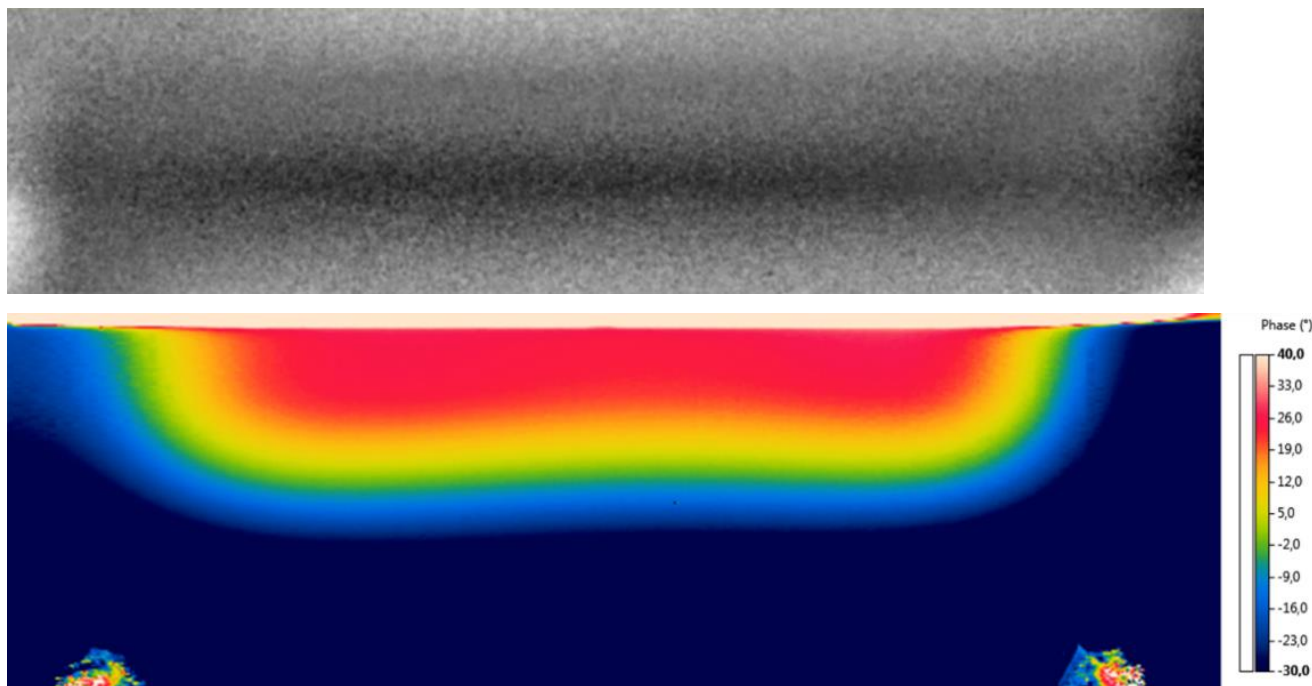

Fig. 6. Shearogram and thermogram of a defect-free testing sample, structural bond, excitation of steel 
The adhesive bond in the shearographic image of the reference sample is homogenous. Furthermore, the adhesive seam is bordered by the horizontal dark line, which constitutes the adhesive border (c.f. figure 6 above). The phase image of this thermographic measurement also shows a homogenous phase progression above the bonding seam (c.f. figure 6 below).

The defect type missing adhesive could clearly be detected both via shearography and thermography (c.f. figure 7). However, this defect was much more difficult to detect when the aluminum substrate was stimulated. The defect missing adhesive is characterized by local changes in stiffness which occur at the boundary to the defect. Therefore, these are easy to detect via shearography. Furthermore, the assumption is that the thermal conductivity from the primarily inductively heated lower substrate to the upper is reduced locally and can then be detected. As a result, these defects are also easy to detect using thermography.
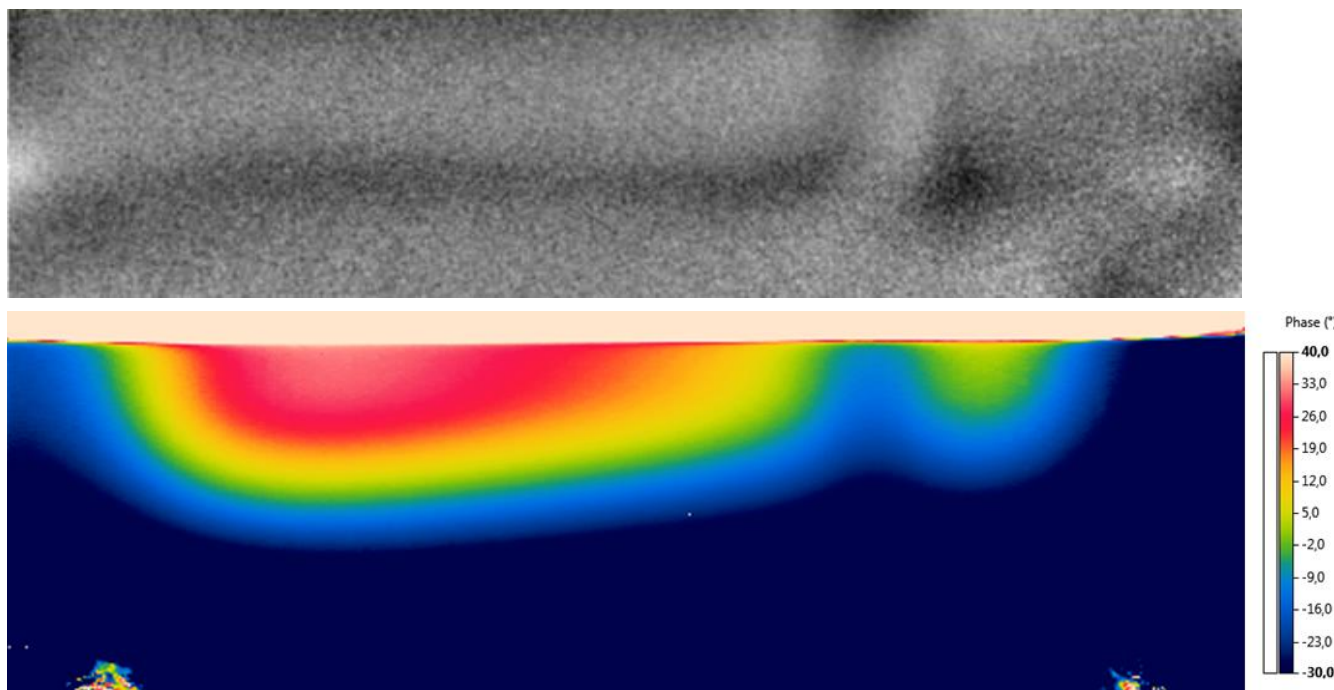

Fig. 7. Shearogram and thermogram of a testing sample with missing adhesive, structural bond, excitation of steel

Voids could - analogous to the defect missing adhesive - also be detected with both methods, independently from which side was stimulated (c.f. figure 8). Voids reduced both stiffness and thermal conductivity of the adhesive on a local level.
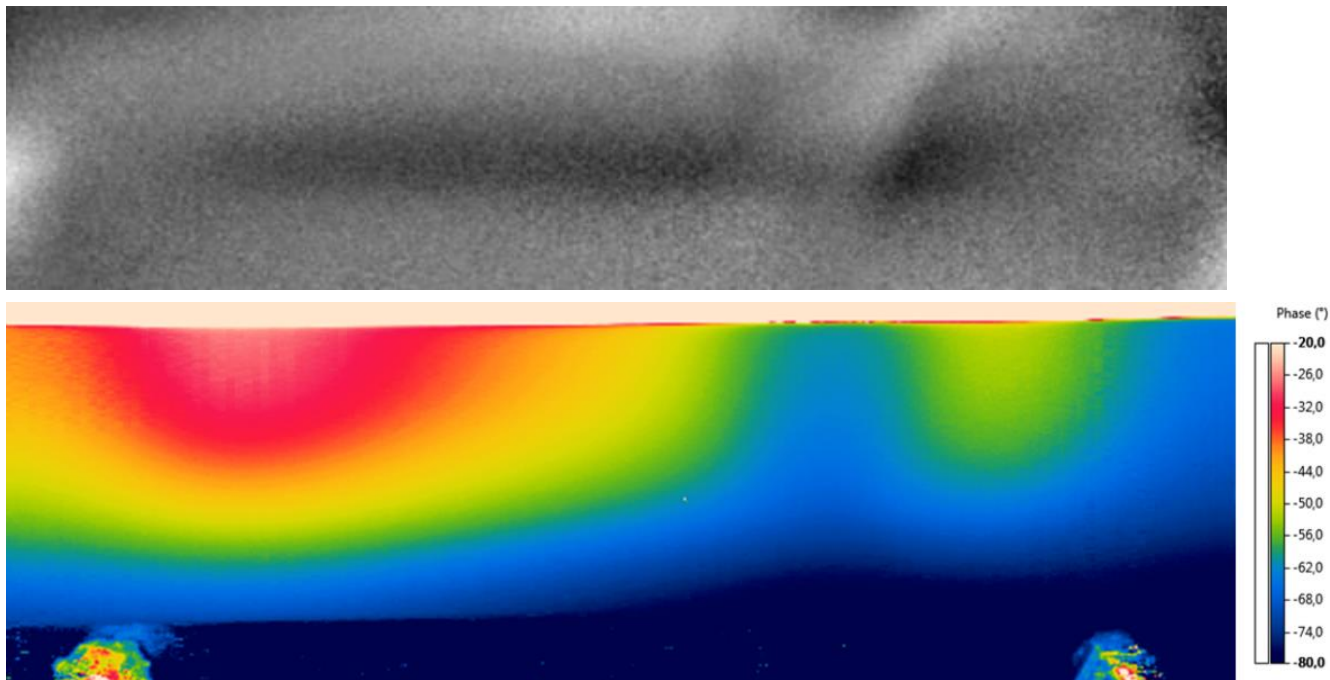

Fig. 8. Shearogram and thermogram of a testing sample with voids, structural bond, excitation of steel

Irregular thickness of the adhesive layer could only be detected with thermography. However, the excitation side had no significant influence on the detection of this defect. The change in the phase angle along the continuously increasing layer of adhesive could be detected easily (c.f. figure 9 below). A detection of the irregular thickness of the adhesive was not possible with shearography from any side of the sample; at least not with the parameters chosen for this testing series (c.f. figure 9 above). A continuous change of stiffness along the shearing angle prevented the detection of this kind of defect. 

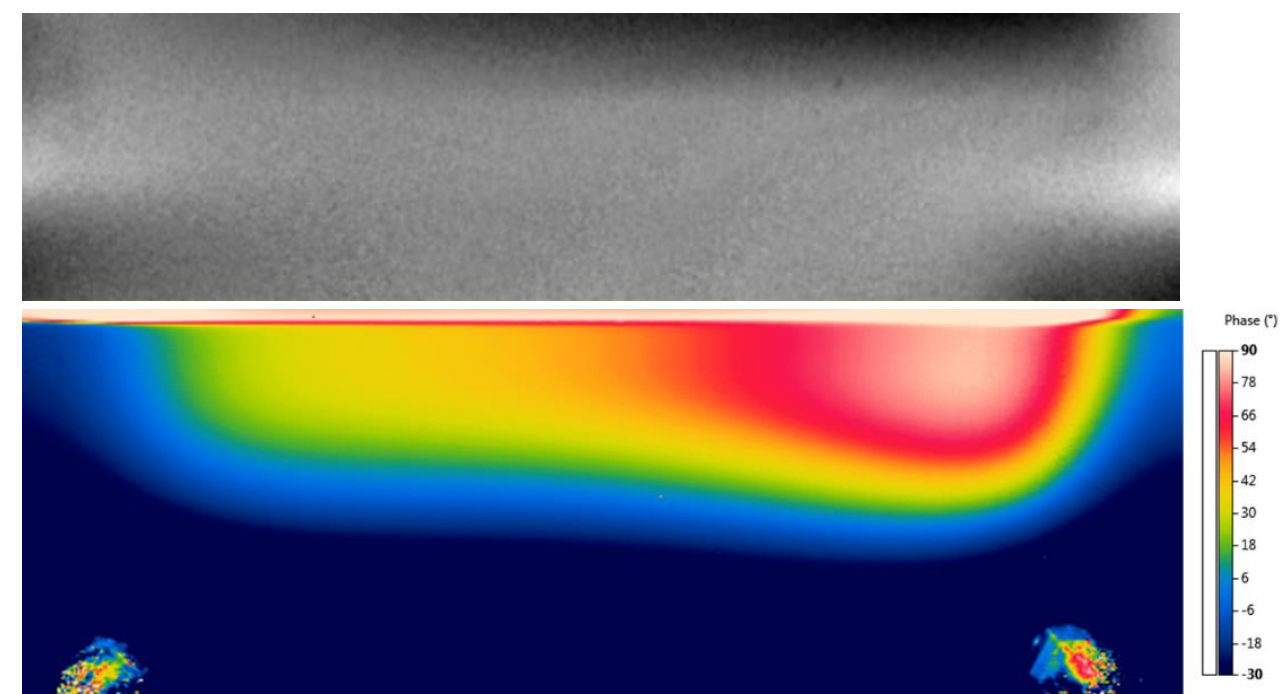

Fig. 9. Shearogram and thermogram of a testing sample with an irregular thickness of the adhesive, structural bond, excitation of steel

The defect type inhomogeneous stirring of adhesive components could be detected clearly from both sides of excitation using shearography. The local disadvantageous relation of resin to curing agent caused detectable changes in stiffness in the adhesive seam, which could then be identified using shearography (c.f. figure 10 above). However, this type of defect was not detectable with thermography. The deviances in thermal conductivity of both adhesive components and their mixture were not large enough to cause a detectable change in the phase angle (c.f. figure 10 below).
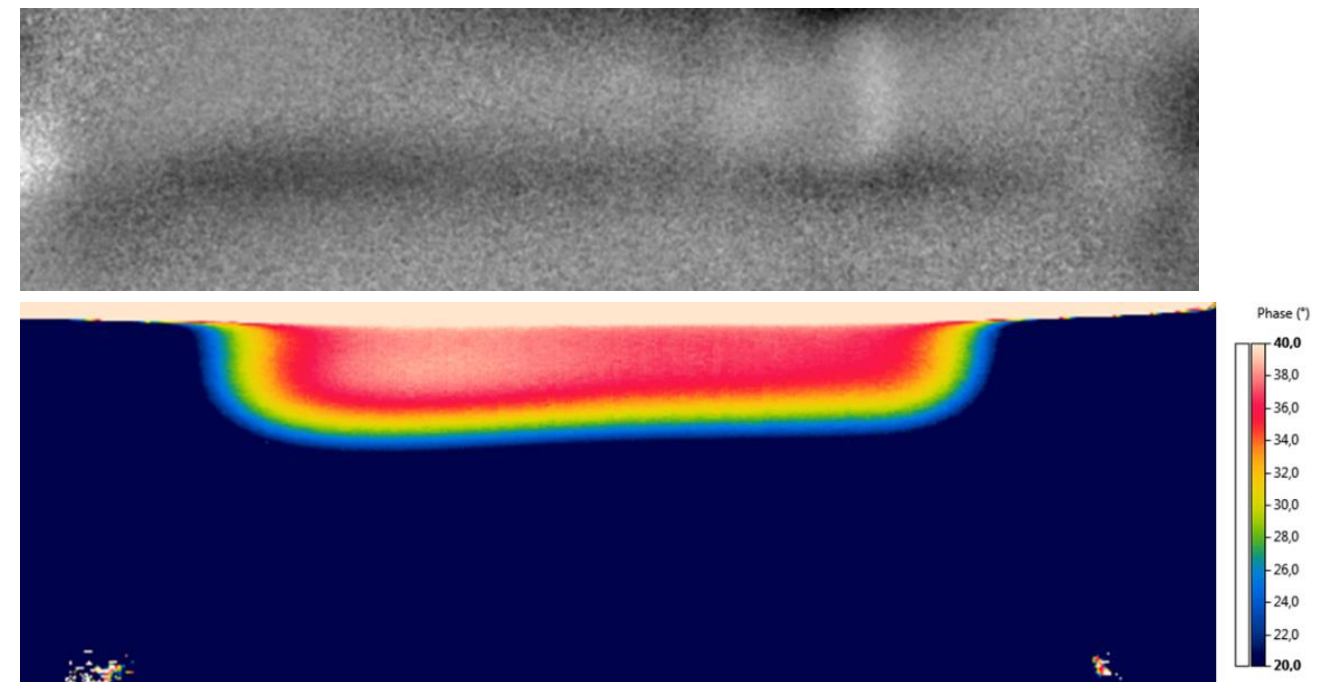

Fig. 10. Shearogram and thermogram of a testing sample with inhomogeneous stirring of adhesive components, structural bond, excitation of steel

The type of defect incorrect mixing ratio could be detected easily with shearography. Which side of the sample was excited had no significant influence on the detection of the defect. The missing resin or curing agent reduced the stiffness significantly (c.f. figure 11 above). However, this type of defect could only be detected in the thermographic images when the aluminum substrate was stimulated after specifically adapting the color scale. A defect detection when stimulating the steel side could not be realized through thermography. Here, the differences in the thermal conductivity of the adhesive and the individual components were too low to be detected when examining the aluminum substrate (c.f. figure 11 below). 

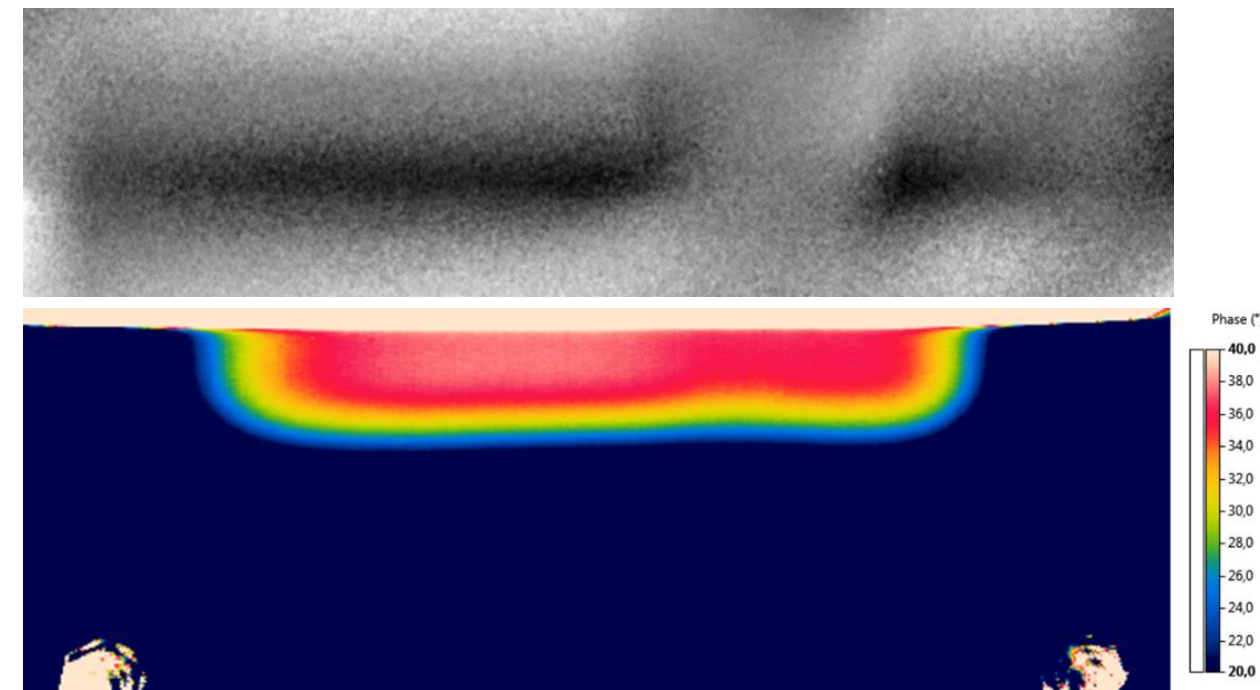

Fig. 11. Shearogram and thermogram of a testing sample with incorrect mixing ratio, structural bond, excitation of steel

Delamination could be detected equally well both with shearography and thermography, no matter which substrate was stimulated (c.f. figure 12). As the delamination prevented the formation of the bond, the stiffness decreased in the area of the defect. Thus, increased deformations could be visualized through inductive excitation in the shearogram. The detachment of the adhesive from the substrate reduced the thermal conductivity between adhesive and substrate which then lead to an inhomogeneous heating of the examined surface of the substrate.
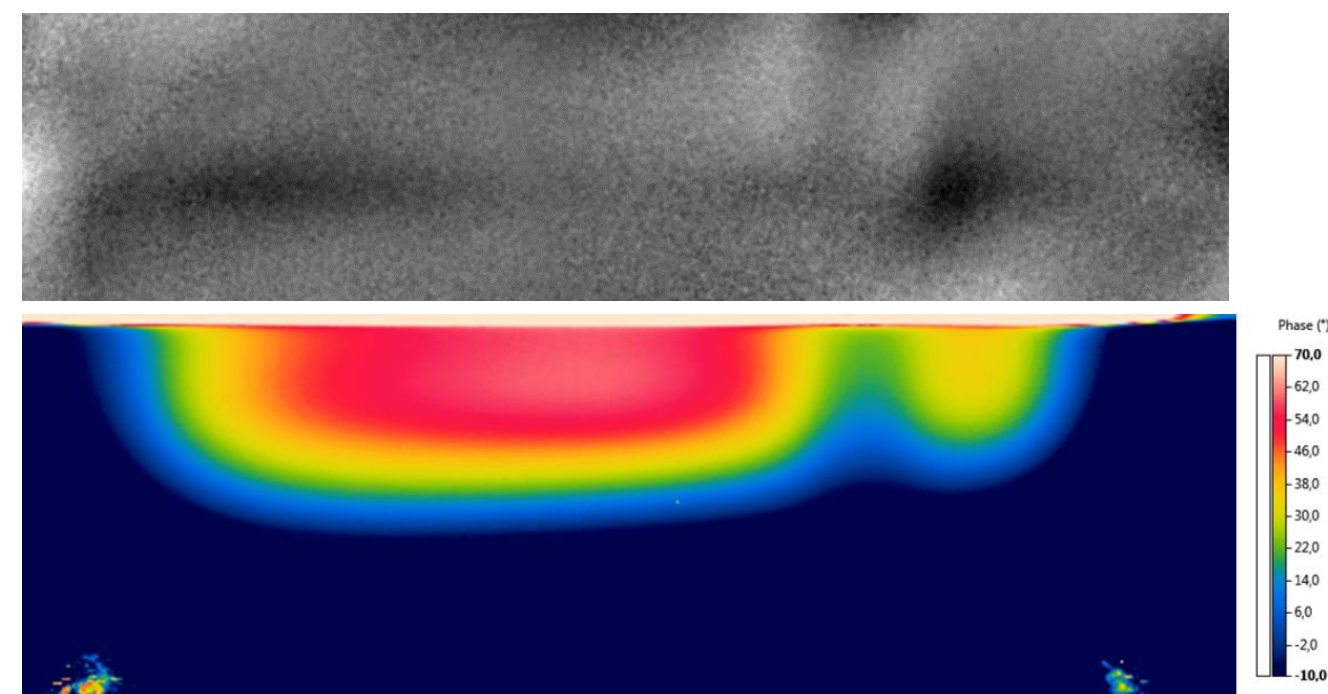

Fig. 12. Shearogram and thermogram of the testing sample with delamination, structural bond, excitation of steel

The defect kissing bond could be detected from both sides of the sample with shearography, however not as clearly as the other types of defects. The shearogram, which allows indirect conclusions as regards the stiffness, is shown in figure 13 (above). In contrast, an uneven heating where the adhesive and the substrate do not touch due to delamination cannot be detected in the thermogram. This leads to the conclusion that the thermal conductivity of the defect is the same as that of an intact bond (c.f. figure 13 below). 

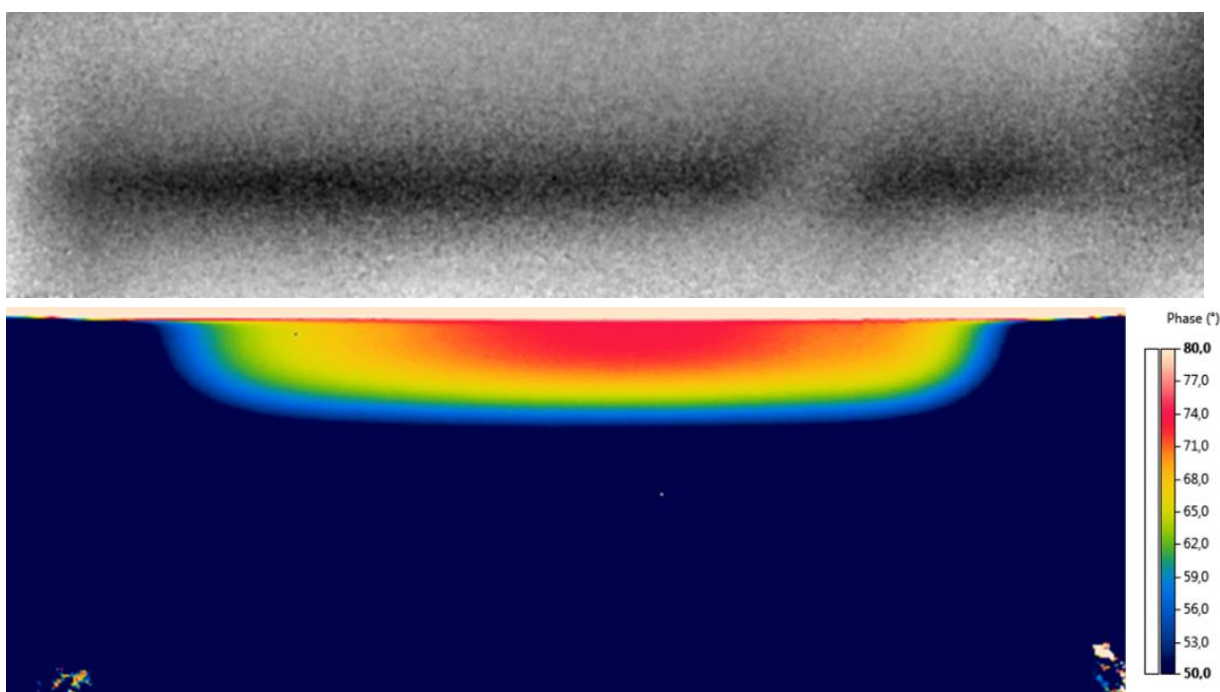

Fig. 13. Shearogram and thermogram of a testing sample with a kissing bond, structural bond, excitation of the steel

\section{Detection of Defects on Application-Oriented Testing Samples via Inductively Induced Shearography and Thermography}

The pulse width modulation (PWM) for shearographic testing of the hat profile was $400 \%$. The most significant shearograms were generated when the induction period was $0.2 \mathrm{~s}$. In contrast, the induction period for thermographic measurements was $0.1 \mathrm{~s}$ with a PWM of $525 \%$. The induction frequency for both testing methods was $12.5 \mathrm{kHz}$.

Figure 14 shows shearograms with defects in the aluminum profile. These allow the conclusion that all inserted defects could be detected clearly - with the exception of the defect kissing bonds.

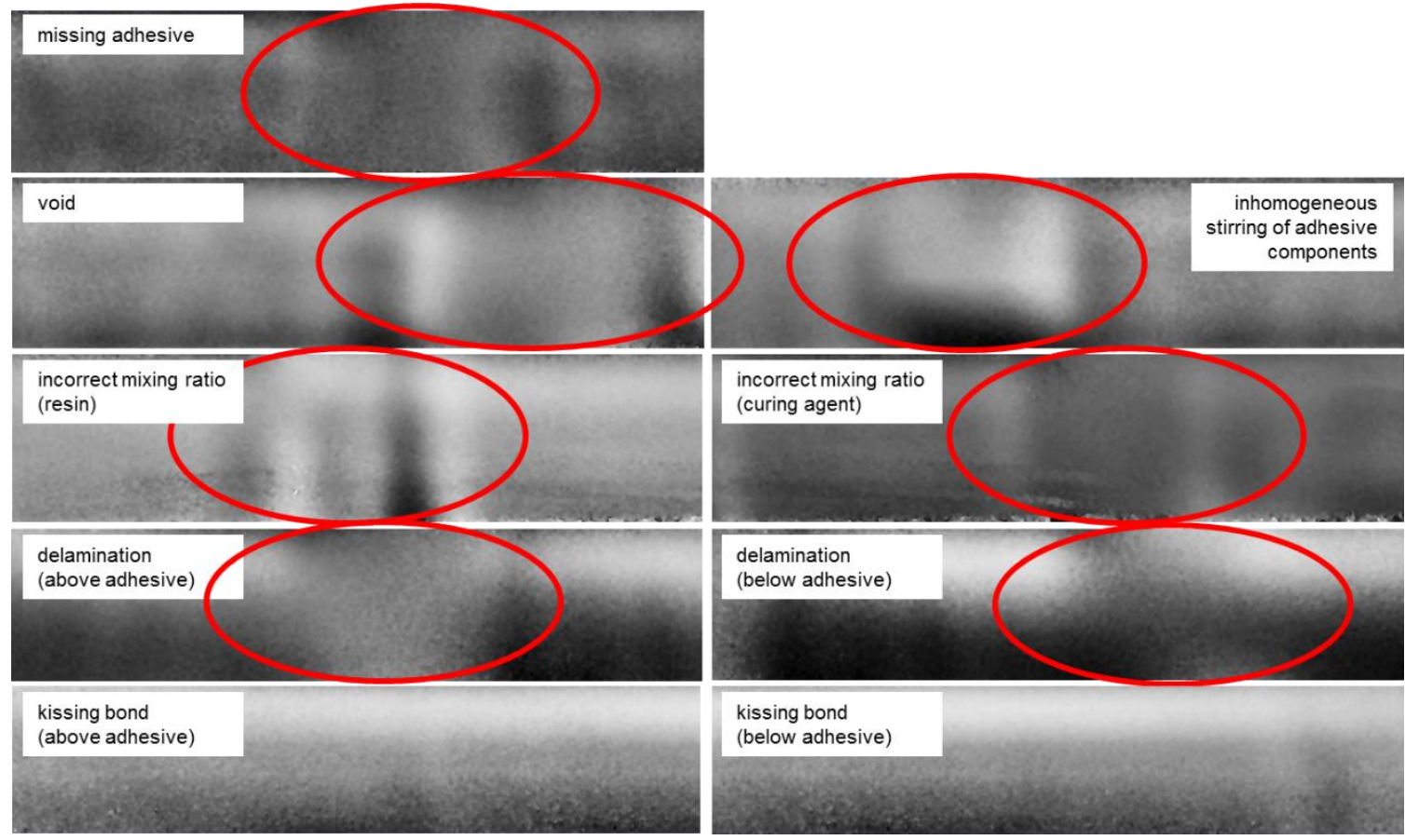

Fig. 14. Shearograms of inserted defects of the application-oriented testing sample

Figure 15 presents the results of the thermographic measurements. The defects missing adhesive, voids, inhomogeneous mixing and delamination were easily detectable. The defects wrong mixing ratio and kissing bonds were superimposed by the unintentionally inserted defect irregular thickness of the adhesive layer (c.f. figure 9); this obstructed the detection of the other defects (wrong mixing ratio, only resin in adhesive; kissing bond below) or even prevented a definite detection (wrong mixing ratio, only hardener in adhesive, kissing bond above). 

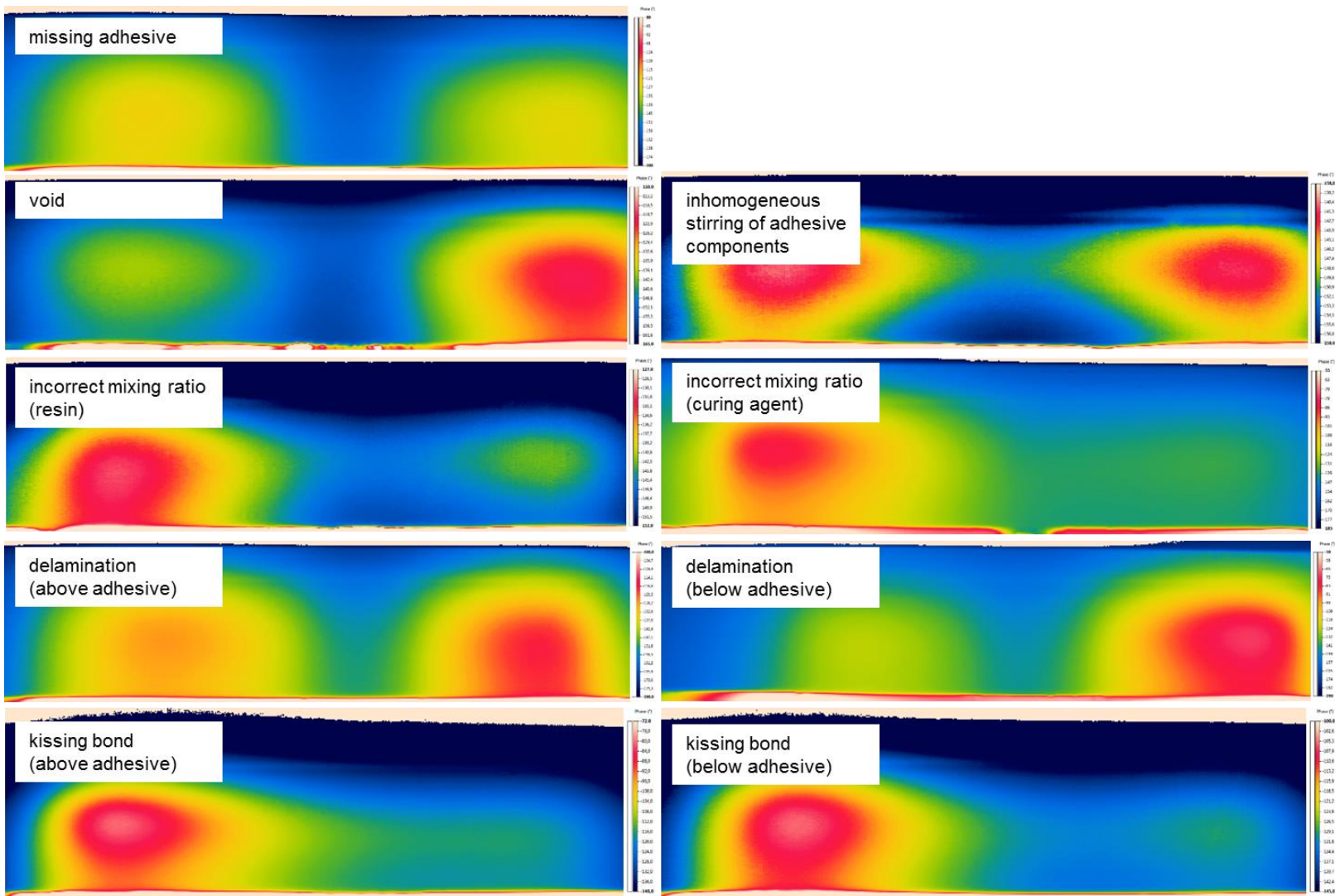

(below adhesive)

Fig. 15. Thermograms of inserted defects of the application-oriented specimen

\section{Conclusion}

This study aimed to introduce and compare defect detection with the non-destructive testing methods shearography and thermography. The testing objects were ideal testing samples and application-orientated samples for industrial purposes. For the examinations, inductive excitation was used for thermal stimulation. Defects such as voids, delamination and kissing bonds were specifically inserted into steel-aluminum testing samples. A high-strength structural adhesive was used for bonding. The results of the detection of defects in the ideal testing samples are shown in table 1 , in dependence to the specific substrate, which was stimulated, and those for application-oriented specimen in table 2. Although most defects could be detected, it was extremely difficult to identify the present type of defect.

In sum, both methods showed similarly good results for structural bonds. Thermography enabled a clear detection of an irregular thickness of the adhesive layer in the ideal testing sample, whilst shearography did not suffice for these testing parameters. Here, the question remains whether a different shearing angle could enable a detection. However, shearography detected the defects inhomogeneous stirring of adhesive components, incorrect mixing ratio and kissing bonds which thermography could not or only hardly make visible for this type of sample.

Table 1. Comparison of defect detection with inductively excited shearography and thermography in the ideal testing sample

\begin{tabular}{|l|c|c|c|c|}
\hline & \multicolumn{2}{c|}{ Shearography } & \multicolumn{2}{c|}{ Thermography } \\
\hline Type of Defect I Side of Stimulation & Aluminum & Steel & Aluminum & Steel \\
\hline Missing adhesive & + & 0 & + & + \\
\hline Void & + & + & + & + \\
\hline Irregular thickness of adhesive & -- & -- & + & + \\
\hline Inhomogeneous stirring of adhesive components & + & + & --- & --- \\
\hline Incorrect mixing ratio & + & + & 0 & --- \\
\hline Delamination & + & + & + & + \\
\hline Kissing Bond & 0 & 0 & --- & -- \\
Detectability: & bad 0 & \multicolumn{2}{|c|}{ no detection --- }
\end{tabular}


With the exception of kissing bonds, all defects could be detected in application-oriented testing samples with shearography. Here, the defects inhomogeneous mixing and wrong mixing ration - flaws that result from deviances in the dosage of the adhesive components - could be found most easily. Thermographic measurements were especially productive for the defects missing adhesive, voids, inhomogeneous mixing and delamination. Wrong mixing ratio (resin component) and kissing bonds (between counterpart sheet and adhesive) could also be detected.

Table 2. Comparison of defect detection with inductively excited shearography and thermography in applicationoriented testing samples

\begin{tabular}{|l|c|c|}
\hline Type of Defect & Shearography & Thermography \\
\hline Missing adhesive & + & + \\
\hline Void & + & + \\
\hline Inhomogeneous stirring of adhesive components & + & + \\
\hline Incorrect mixing ratio (missing curing agent) & + & 0 \\
\hline Incorrect mixing ratio (missing resin) & + & -- \\
\hline Delamination (above) & + & + \\
\hline Delamination (below) & 0 & + \\
\hline Kissing Bond (above) & --- & --- \\
\hline Kissing Bond (below) & --- & 0 \\
\hline Detectability: & good $+\quad 0 \quad$ no detection ---
\end{tabular}

A comparison of these results shows that both methods identify different defects in a bond due to their distinct physical measuring parameters. The respective methods suit different types of defects. Furthermore, the results show that a combination of both methods in one set-up is profitable for the non-destructive testing of adhesive bonds. By combining both methods simultaneously, a measurement system could be developed which meets the requirements of industrial process control (e.g. short measurement period) and offers a significantly higher rate of defect detection (especially for kissing bonds).

\section{Acknowledgements}

The IGF project No: 18.709 N / DVS-No: 08.095 of the research association "Forschungsvereinigung Schweißen und verwandte Verfahren e. V." of the DVS, Aachener Str. 172, 40223 Düsseldorf, Germany, was supported by the AiF within the scope of a program for the fostering of the industrial collective research (IGF) by the Federal Ministry of Economic Affairs and Energy due to a resolution of the German Bundestag. We appreciate this support very much. Furthermore, we would like to thank the committee which accompanied the project for their constructive support during in this project.

\section{REFERENCES}

[1] Adams R., "Adhesive bonding. Science, technology and applications". CRC Press, Boca Raton, Florida, 2005.

[2] Silva L., Öchsner A., Adams R., "Handbook of adhesion technology". Springer-Verlag, Berlin, London, 2011.

[3] Steinchen W., Yang L., "Digital shearography. Theory and application of digital Speckle pattern shearing interferometry". SPIE Optical Engineering Press, Bellingham, Wash, 2003.

[4] Hung Y., Chen Y., Ng S., Liu L., Huang Y., Luk B., Ip R., Wu C., Chung P., "Review and comparison of shearography and active thermography for nondestructive evaluation". Materials Science and Engineering: R: Reports, vol. 64, pp. 73-112, 2009.

[5] Wilhelm T., Hinnen M., Schmidt W., Montnacher J., Verl A., "Non-destructive testing of adhesive joints. Production-ready method of quantifying adhesion properties". adhesion ADHESIVES \& SEALANTS, no 4 (2010), pp. 20-24 2010.

[6] Hung M., Chen Y., Ng S., Shepard S., Hou Y., Lhota J., "Review and comparison of shearography and pulsed thermography for adhesive bond evaluation". Optical Engineering, vol. 46, no 5, pp. 051007-1 - 051007-16, 2007.

[7] Kryukov I., Böhm S., "Prospects and limitations of eddy current shearography for non-destructive testing of adhesively bonded structural joints". The Journal of Adhesion, 2018.

[8] Meola C., Carlomagno G., Giorleo G., "Using infrared thermography to analyze substrate and adhesive effects in bonded structures". Journal of Adhesion Science and Technology, vol. 18, no 6, pp. 617-634, 2004.

[9] Rudnev V., Loveless D., Cook R., "Handbook of Induction Heating". Second Edition, CRC Press, Boca Raton, Florida, 2017. 